\section{Dificuldades na execução das diretrizes do Programa de Vigilância e Controle da Leishmaniose Visceral em grandes municípios brasileiros}

\author{
Difficulties in implementing the guidelines of the \\ Brazilian Visceral Leishmaniasis Control Program \\ in large cities
}

Las dificultades en la aplicación de las directrices del Programa Brasileño de Control de la Leishmaniasis Visceral en grandes

ciudades brasileñas

\section{Resumo}

A expansão das áreas de transmissão de leishmaniose visceral (LV) traz questionamentos sobre as estratégias de controle empregadas no Brasil. As diretrizes do Programa de Vigilância e Controle da Leishmaniose Visceral (PVCLV) estão centradas na diminuição da morbidade e letalidade, controle do reservatório e dos vetores e na educação em saúde. Este artigo buscou avaliar as dificuldades na execução das ações preconizadas pelo PVCLV, segundo os seus coordenadores em municípios brasileiros de grande porte com transmissão canina elou humana (Campinas, Bauru, Goiânia, Campo Grande, Fortaleza e Belo Horizonte). Foram realizadas entrevistas semiestruturadas, sendo os principais problemas identificados: descontinuidade das atividades de controle, resistência dos proprietários de cães com indicação de eutanásia e baixa cobertura do controle químico. Como conclusão, as entrevistas mostraram que inúmeros fatores impedem o cumprimento das atividades propostas pelo PVCLV, ficando clara a necessidade de reavaliação da política brasileira de controle de LV.

Leishmaniose Visceral; Prevenção de Doenças; Planos e Programas de Saúde
Andrea Paula Bruno von Zuben 1 Maria Rita Donalísio 1

\author{
Correspondência \\ A. P. B. von Zuben \\ Rua do Joá 310, Campinas, $S P$ \\ 131014-116, Brasil. \\ andreavonzuben@uol.com.br
}




\section{Introdução}

Causada no Brasil pelo protozoário Leishmania (L.) infantum e transmitida predominantemente pelo vetor Lutzomyia longipalpis, a leishimaniose visceral (LV) apresenta como seu principal reservatório no ambiente urbano o cão doméstico, tanto no Brasil como em outros países das Américas 1,2.

A cada ano, quase dois milhões de novos casos dessa importante zoonose são registrados no mundo, segundo estimativas da Organização Mundial da Saúde (OMS). Cerca de 90\% dos casos da América Latina ocorrem no Brasil, com quase três mil pessoas sendo infectadas pela doença anualmente 2,3,4. A doença deixa de ter caráter rural e passa a se expandir e se tornar de caráter urbano no país a partir da década de 1980, com o registro de transmissão em áreas urbanizadas em cidades de maior dimensão. Anteriormente restrita ao Nordeste, dissemina-se por várias cidades do Norte, Centro-oeste e Sudeste, causando epidemias urbanas e aumento de incidência e letalidade 2,5,6.

Para conter esse avanço territorial e diminuir a morbidade e letalidade do agravo, o Ministério da Saúde publicou no ano de 2006 o Programa de Vigilância e Controle da Leishmaniose Visceral (PVCLV) 7, com medidas baseadas no diagnóstico e tratamento precoce dos casos humanos, redução da população de flebotomíneos, eliminação de reservatórios e atividades de educação em saúde. Ainda conforme o PVCLV, as ações de prevenção e controle adotadas pelos municípios devem estar baseadas em análises epidemiológicas fundamentadas na estratificação por risco e o cumprimento destas deve ser realizado de forma integrada, alertando ao fato de que nenhuma das ações isoladamente é capaz de prevenir e controlar o agravo em sua totalidade.

No entanto, apesar dos esforços, identificase a expansão dos casos autóctones humanos e das áreas endêmicas urbanas de LV no Brasil 5,6. Assim, o objetivo deste trabalho é analisar insuficiências e dificuldades encontradas para a execução de medidas de controle da doença propostas no PVCLV, em grandes centros urbanos de diferentes regiões do país, de acordo com a opinião dos coordenadores municipais deste programa.

\section{Método}

Foram realizadas entrevistas com gestores municipais de programas de controle de LV de seis municípios brasileiros, sendo a escolha dos coordenadores de programa participantes feita de forma intencional, considerando o tamanho do município, a presença de transmissão humana e/ou canina de LV, o fato de pertencerem a diferentes regiões do país e diferentes estratos epidemiológicos.

Campo Grande (Mato Grosso do Sul), Fortaleza (Ceará) e Belo Horizonte (Minas Gerais) foram escolhidos por apresentarem o maior número de casos humanos confirmados entre todos os municípios brasileiros 7,8. Bauru foi selecionado por ser o município com o maior número de casos humanos do Estado de São Paulo 7,9. Goiânia (Goiás) e Campinas (São Paulo) foram elencados por terem semelhança no número de habitantes e apresentarem somente transmissão canina 10,11 .

As entrevistas foram feitas entre os anos de 2012 e 2013 por meio de questionários semiestruturados com o seguinte formato: questões a serem respondidas com alternativas prédeterminadas e questões abertas para que o entrevistado expusesse os seus pontos de vista (Figura 1). As respostas foram agrupadas e analisadas segundo categorias temáticas destacadas no questionário.

As entrevistas com os coordenadores de Campinas, Goiânia, Campo Grande e Fortaleza foram realizadas por uma das autoras do presente artigo, e as de Belo Horizonte e Bauru foram enviadas por e-mail e respondidas pelos próprios participantes.

O roteiro previu a abordagem de três aspectos dos programas municipais:

(1) Caracterização epidemiológica dos municípios - número de habitantes; início da transmissão autóctone de LV; nome, cargo e tempo de atuação do coordenador municipal no Programa, composição, número e tempo de existência das equipes. Dados epidemiológicos foram obtidos, de forma complementar, no banco de dados do Departamento de Informática do SUS (DATASUS);

(2) Diferenças entre diretrizes federais e realidades municipais e principais dificuldades encontradas;

(3) Dificuldades na execução das diretrizes de prevenção e controle contidas no PVCLV em cada município - medidas referentes ao vetor e à educação em saúde e ao reservatório canino, priorizando questões sobre eutanásia.

Esta pesquisa foi aprovada pelo Comitê de Ética em Pesquisa da Faculdade de Ciências Médicas da Universidade Estadual de Campinas (CAAE: 01196312.6.0000.5404). Foram tomados cuidados especiais na análise do material para evitar a identificação dos programas municipais. 
Figura 1

Entrevista do Programa de Vigilância e Controle da Leishmaniose Visceral (PVCLV) em municípios com transmissão.

\section{RESPONSÁVEL PELO PROGRAMA}

Município:

Habitantes:

Quanto tempo no Programa:

1) Existe uma equipe específica para realizar as ações do PLV?

Sim ( ) Não ( ) Há quanto tempo? (anos)

2) A equipe é exclusivamente municipal? Sim ( ) Não ( )

3) Quantos técnicos (nível superior/operacional/de campo) suficientes? Sim ( ) Não ( )

4) Tem deslocamento de equipe do âmbito estadual ou federal para a realização de atividades de campo no município? Sim ( ) Não ( )

1) É realizada eutanásia de cães reagentes? Sim ( ) Não ( )

2) Somente é realizada a eutanásia com o consentimento do proprietário? Sim ( ) Não ( )

3) Há lei que ampare a eutanásia sem consentimento no município? Sim ( ) Não ( )

4) Qual o critério para a eutanásia?

( ) 2 sorologias positivas

( ) 1 sorologia positiva

( ) Animal sintomático sem sorologia

( ) Outro exame. Qual?

5) Há resistência por parte dos proprietários para a realização de eutanásia?

( ) Em pequena \% da população $(<10 \%)$

( ) Entre 10 e $50 \%$

( ) Mais de $50 \%$
Nome:

Cargo:

Contato - Telefone/Email:

5) Tem autonomia municipal para a tomada de decisões sobre estratégias de controle LV?

Sim ( ) Não ( )

6) A equipe faz análise epidemiológica para avaliar ações do Programa? Sim ( ) Não ( )

7) Há diferenças entre os Programas municipal e o preconizado pelo Ministério? Sim ( ) Não ( ) Se sim. Quais?

8) Qual(is), na sua opinião, é(são) a(as) principal(is) dificuldade(s) do

Programa?

\section{RESERVATÓRIO CANINO}

6) Quanto tempo demora entre o resultado reagente e a eutanásia?
( ) Menos de 1 semana
( ) Entre 1 semana e 1 mês
( ) Mais de 1 mês

7) Qual o motivo, em sua opinião, da ocorrência de recusa?
( ) Afeto ao animal
( ) Descrédito no Programa
( ) Animais pouco sintomáticos/assintomáticos
( ) Desconhecimento sobre o risco da transmissão do animal para o humano
( ) Interferência de ONG protetora de animais
( ) Interferência do médico veterinário particular
( ) exames laboratoriais pouco confiáveis
( ) Outros. Especifique:

\section{EDUCAÇÃO EM SAÚDE}

1) Na sua opinião, qual a porcentagem da população que adere às medidas propostas pelo Programa?

( ) Em pequena $\%$ da população $(<10 \%)$

( ) Entre 10 e $50 \%$

( ) Mais de $50 \%$

2) Qual é a forma de abordagem da população nas ações educativas realizadas pelo Programa?

( ) Palestras

( ) Folders/material educativo

( ) Meios de comunicação/imprensa

( ) Trabalho educativo casa a casa

( ) Outros. Quais?

3) Na sua opinião, qual(is) é(são) a(s) principal(is) dificuldade(s) das ações educativas realizadas pelo Programa?

Teve algum problema com veterinários particulares?

Sim ( ) Não ( ) Quais?

Teve algum problema com sociedade protetora dos animais?

Sim ( ) Não ( ) Quais?

Observações: 


\section{Resultados e discussão}

\section{Caracterização epidemiológica}

dos municípios

Todos os municípios estudados são considerados de grande porte, sendo o menor deles Bauru, com 343.937 habitantes, e o maior Fortaleza, com 2.551.806 habitantes. Os demais tinham o seguinte número de habitantes: Belo Horizonte - 2.375.444, Goiânia - 1.333.767, Campinas 1.144.862 e Campo Grande - 786.797.

A LV é um dos maiores problemas de saúde pública enfrentados em Campo Grande, pois desde os primeiros relatos de casos em 2002 a incidência se manteve elevada, com altas taxas de letalidade 12,13. Considerando-se o período entre 2011 e 2013, Campo Grande apresentou o maior coeficiente de incidência entre os quatro municípios com transmissão humana estudados. No entanto, apesar da alta incidência, a letalidade pela doença foi a menor dentre estes (Tabela 1).

Em Belo Horizonte, o relato do primeiro caso de LV foi no ano de 1994 e desde então a doença expandiu no município, com ampla distribuição espacial. Alguns estudos indicaram tendência crescente dos coeficientes de incidência e letalidade em humanos e de prevalência em cães no município 14,15. Nos anos avaliados, Belo Horizonte, apesar de ter apresentado o menor coeficiente de incidência, registrou a mais alta letalidade entre os quatro municípios estudados (Tabela 1).

Em Fortaleza, a doença é endêmica em humanos desde 1995, com acentuada expansão geográfica de 1999 a 2007, ocorrendo em focos distribuídos em toda a cidade, com alta incidência humana e canina e alta letalidade. A LV no Município de Fortaleza ainda está em processo de urbanização, com número de casos crescente ao longo dos anos 16,17. Entre 2011 e 2013, houve manutenção das taxas de letalidade e tendência de estabilização dos coeficientes de incidência (Tabela 1).

Em Bauru, a LV foi notificada pela primeira vez em 2003 e, apesar da implantação de controle e prevenção, a doença continua a se expandir na área urbana com aumento do número de casos, bem como da letalidade 18,19. Embora em números absolutos seja a cidade com menos casos entre as estudadas, tem coeficientes de incidência mais altos que Fortaleza e Belo Horizonte. No entanto, registra a mais baixa letalidade entre os quatro municípios comparados, com queda deste coeficiente ano a ano, considerando-se o período de 2011 a 2013 (Tabela 1).

A Tabela 1 mostra o número de casos, coeficiente de incidência e letalidade da LV nos municípios com transmissão humana.

Em Campinas e Goiânia há somente transmissão canina de LV, que ocorre desde 2009 em Campinas 11 e 2011 em Goiânia 10. Ambos os municípios, na ocasião das entrevistas, registravam transmissão focal, ou seja, restrita geograficamente a uma localidade. Sendo assim, a meta nesses municípios segundo o PVCLV é desencadear as ações visando, principalmente, à prevenção da ocorrência de casos e óbitos humanos.

Dos seis coordenadores entrevistados em 2013, cinco eram médicos veterinários e um biólogo. Os demais componentes das equipes eram técnicos de nível superior e operacionais de campo.

Nos municípios onde há transmissão canina e humana, o número de operacionais de campo variou de 22 a 170 e de nível superior de 2 a 14 técnicos. Nos municípios em que há somente transmissão canina, o número de trabalhadores operacionais de campo variou de 10 a 30 e o de técnicos de nível superior entre 5 e 9 profissionais. Em um município havia servidores estaduais e federais compondo a equipe.

Em todos os municípios, a estruturação de equipes de LV variou entre 2 e 18 anos, e foram coincidentes com o registro de autoctonia, não

Tabela 1

Leishmaniose visceral: casos e óbitos confirmados, coeficiente de incidência e letalidade por município. Brasil, 2011-2013.

\begin{tabular}{|c|c|c|c|c|c|c|c|c|c|c|c|c|}
\hline \multirow[t]{2}{*}{ Município } & \multicolumn{3}{|c|}{ Casos (n) } & \multicolumn{3}{|c|}{$\begin{array}{c}\text { Coeficiente de } \\
\text { incidência (por } \\
100.000 \text { habitantes) }\end{array}$} & \multicolumn{3}{|c|}{ Óbitos (n) } & \multicolumn{3}{|c|}{ Letalidade (\%) } \\
\hline & 2011 & 2012 & 2013 & 2011 & 2012 & 2013 & 2011 & 2012 & 2013 & 2011 & 2012 & 2013 \\
\hline Bauru & 39 & 43 & 30 & 11,3 & 12,5 & 8,7 & 4 & 2 & 1 & 10,3 & 4,7 & 3,3 \\
\hline Belo Horizonte & 108 & 70 & 66 & 4,5 & 2,9 & 2,8 & 15 & 12 & 8 & 13,9 & 17,1 & 12,1 \\
\hline Campo Grande & 150 & 213 & 170 & 19,1 & 27,1 & 21,6 & 3 & 13 & 12 & 2,0 & 6,1 & 7,1 \\
\hline Fortaleza & 249 & 155 & 155 & 9,8 & 6,1 & 6,1 & 21 & 13 & 13 & 8,4 & 8,4 & 8,4 \\
\hline
\end{tabular}

Fonte: SINAN-net (Sistema de Informação de Agravos de Notificação), Ministério da Saúde. 
obstante diretrizes do PVCLV que indicam a realização de ações antes do aparecimento de casos humanos ou caninos da doença.

Três dos seis coordenadores entrevistados consideraram insuficientes os recursos humanos, prejudicando o cumprimento das metas do PVCLV. Ao serem questionados sobre os critérios para a contratação de profissionais para o programa, quatro coordenadores citaram que a priorização dada pela Secretaria Municipal de Saúde não coincide, necessariamente, com o tamanho e/ou demanda do município.

Segundo os coordenadores entrevistados, por não haver parâmetros técnicos no programa federal que indiquem categorias profissionais e/ ou número mínimo de agentes operacionais a serem contratados pelos municípios, há dificuldade na negociação por mais recursos humanos nas prefeituras.

\section{Diferenças entre diretrizes federais e realidades municipais e principais dificuldades encontradas}

Os gestores municipais foram questionados sobre a execução das medidas de prevenção e controle de LV com o objetivo de comparar com as preconizadas pelo programa federal. Foi solicitada a avaliação dos gestores sobre as dificuldades encontradas.

As propostas existentes no PVCLV buscam contribuir com as secretarias municipais de saúde na organização e execução das atividades de prevenção e controle de LV 11, com autonomia do âmbito local para a tomada de decisões mais adequadas à realidade da região. Por isso, o questionário buscou entender essa relativa independência. Cinco coordenadores relataram ter autonomia relativa, uma vez que as atividades do PVCLV não permitem muita adequação à realidade local. Já um dos coordenadores afirmou não ter qualquer autonomia para a tomada de decisão que diferisse do contido no programa federal.

Apesar de relatarem pouca autonomia, cinco dos seis coordenadores de programas municipais mencionaram haver divergência entre o preconizado pelo PVCLV e o que é efetivamente realizado no município. Como principal justificativa, todos relataram dificuldades na execução do trabalho de campo preconizado pelo PVCLV, pela complexidade do controle da doença. As principais dificuldades apontadas foram agrupadas e listadas a seguir:

(1) Recusa da população (citada por todos). Relato de impedimento da entrada de técnicos nas residências para controle químico e para o controle do reservatório canino.
(2) Eutanásia como a principal medida indicada ao reservatório doméstico (citada por todos). Registrou-se grande resistência por parte dos proprietários dos animais, bem como da comunidade, como mostra a fala de um dos coordenadores: "a eutanásia do cão da família dificulta o bom relacionamento dos técnicos do programa com a comunidade envolvida com o problema". Nesse contexto, três coordenadores citaram que há interferência judicial com liminares de proprietários de cães contra a eutanásia, dificultando ainda mais esta atividade.

(3) Custo muito alto (4 citações). Relato de que as ações preconizadas pelo programa requerem muito investimento financeiro e os recursos advindos do Ministério da Saúde e complementados pelos municípios são insuficientes. De forma que há descontinuidade de atividades por falta de recursos materiais, financeiros e humanos, em especial durante epidemias de dengue. Demora na aquisição de insumos por conta dos trâmites burocráticos das prefeituras também colaboram na escassez de materiais, particularmente para a eutanásia canina (falta de exames laboratoriais, de agulhas, seringas, anestésicos, entre outras) e controle químico do vetor (falta de inseticidas, por exemplo).

(4) Pouco envolvimento de outros setores das prefeituras (2 citações). Relato de falta de vontade política dos gestores municipais (secretários de saúde e prefeitos) com pouca priorização no controle da LV na prefeitura, tendo como consequência a escassez do envolvimento de outras secretarias no controle da doença.

Vários autores citam dificuldades concernentes à implementação do PVCLV relacionadas à insuficiência de recursos humanos, materiais e financeiros 20,21, levando à descontinuidade das ações 17,22. Essa insuficiência de recursos pode ter entre uma de suas explicações a abrangência de atividades a serem desenvolvidas pela vigilância em saúde no Brasil que compreende, além da vigilância das doenças transmissíveis, doenças e agravos não transmissíveis 23 . De forma que o repasse federal por meio do teto financeiro de epidemiologia e controle de doenças acaba sendo insuficiente para todo o escopo de atuação, havendo disputa por recursos entre os vários programas realizados pelas equipes de vigilâncias municipais.

A interrupção das atividades e/ou a sua realização parcial fragiliza o PVCLV, uma vez que impossibilita o cumprimento de todas as ações preconizadas, podendo ser uma das explicações para o avanço territorial da doença e aumento de morbidade e letalidades da LV. 
Forma de execução das diretrizes de prevenção e controle contidas no PVCLV

Os gestores municipais de programa foram questionados sobre como efetivamente eram executadas as atividades contidas no PVCLV, levando em consideração as fragilidades encontradas. Os resultados e a discussão foram agrupados segundo categorias temáticas para facilitar o entendimento.

\section{- Medidas referentes ao reservatório canino, focando a indicação da eutanásia canina}

Devido à importância do cão como reservatório da LV, o Ministério da Saúde adota no Brasil a eliminação destes animais quando são soropositivos para L. infantum 7 como medida de controle em áreas endêmicas.

Embora preconizada no Brasil, os resultados da eutanásia canina são controversos. Sob o ponto de vista da saúde pública, tem justificativas fundamentadas em alguns fatores: o fato de caninos domésticos serem responsáveis pela dispersão da doença a partir de focos enzoóticos; grande contingente de animais assintomáticos albergando parasitos na derme, com potencial para transmitir a doença; e ainda a constatação de que a doença canina geralmente precede a doença humana 24. Diversos estudos corroboram a tese defendida pelo PVCLV, uma vez que demonstram que a eutanásia em cães sororeagentes reduz o número de animais infectados e assim a prevalência/incidência humana de LV 25 .

Em contrário, uma série de outras pesquisas concluiu que não há correlação espacial entre a incidência acumulada de LV humana com a soroprevalência canina, que há ausência de risco significativo de infecção humana na coabitação com cães, que a eutanásia isoladamente não demonstrou vantagem em reduzir a incidência de LV em seres humanos e que existe a possibilidade do envolvimento de outros reservatórios de infecção de L. infantum, tais como pessoas (particularmente crianças desnutridas que podem transmitir para outras crianças), canídeos silvestres e marsupiais $25,26,27,28$.

Além de controversa do ponto de vista científico, a eutanásia como medida de saúde pública tem pouca eficácia pela rejeição desta atividade por parte da comunidade, como demonstra o percentual de recusa à eutanásia referida pelos coordenadores de programas neste estudo (Tabela 2). Dentre os seis coordenadores entrevistados, somente um deles refere resistência à eutanásia em menos de $10 \%$ da população abordada. E mesmo assim, relata que há heterogeneidade
Tabela 2

Recusa para a realização da eutanásia de cães soropositivos por parte da população, segundo os coordenadores de programa entrevistados em seis municípios brasileiros. Brasil, 2013

\begin{tabular}{lcc}
\hline Recusa & \multicolumn{2}{c}{ Frequência de resposta } \\
& $\mathbf{n}$ & $\%$ \\
\hline Em mais de $50 \%$ & 3 & 50,0 \\
Entre 10 e $50 \%$ & 1 & 16,7 \\
$<10 \%$ & 2 & 33,3 \\
Total & 6 & 100,0 \\
\hline
\end{tabular}

Fonte: questionários semiestruturados.

municipal e que em classes sociais mais abastadas essa recusa costuma ser superior a $50 \%$. É importante relatar que nenhum dos municípios estudados tem lei que ampare a realização de eutanásia sem consentimento do proprietário, pois alegam que tal lei contribuiria para aumentar ainda mais a hostilidade da comunidade ao PVCLV.

A eutanásia animal é tema regido pela Resolução no 1.000, de 11 de maio de 2012, na qual consta que "a eutanásia deve ser indicada quando o bem-estar animal estiver ameaçado e também quando o animal constitui-se ameaça à saúde pública" 29, dando margem tanto para a defesa da vida animal quanto para respaldar a ação da saúde pública. O enfoque individual é claramente identificado na posição de proprietários de animais, de organizações não governamentais (ONGs) e de clínicos veterinários. Esses grupos colocam a doença, o risco e as ações sob um prisma particular e pessoal, sendo a preocupação pautada em fenômenos biológicos individuais que visam à preservação da vida considerando o afeto pelo animal e colocando o cão como membro da família 30 . Por outro lado, o programa de saúde pública age sob a égide do coletivo com enfoque epidemiológico, visando à interrupção da transmissão da doença, pautando a atuação no controle e na prevenção nas populações humanas, e para isto atua com o objetivo de identificar o foco e eliminar o reservatório da doença na coletividade.

Ao serem questionados sobre quais seriam os motivos de haver recusa da população à eutanásia canina, os entrevistados citaram: afeto pelo animal (citado por todos); animais reagentes, porém oligosintomáticos ou assintomáticos (citado por todos); interferência de clínico veterinário particular (citado por todos); interferência 
de ONGs protetoras de animais (citado por 5 dos 6); descrédito no programa (citado por 4 dos 6); exames laboratoriais pouco confiáveis (citado por 4 dos 6); e desconhecimento sobre o risco da transmissão animal para o humano (citado por 3 dos 6).

A limitada acurácia dos métodos diagnósticos utilizados na população canina citada por quatro coordenadores pode ser um fator limitante para a efetividade da eutanásia como medida de controle da LV. Para a indicação de eutanásia, o Ministério da Saúde recomenda dois testes sorológicos reagentes: o Dual Path Platform (DPP) (cuja sensibilidade varia entre $93 \%$ e $100 \%$ e especificidade entre $92 \%$ e $100 \%$ ), que é tecnologia de imunoensaio cromatográfico para testes de diagnóstico rápido como teste de triagem, e ELISA (cuja sensibilidade varia entre $91 \%$ e $97 \%$ e especificidade entre $83 \%$ e $98 \%$ ) como teste confirmatório 31. Exames sorológicos podem gerar tanto resultados falsos positivos como falsos negativos (mantendo cães que podem infectar o vetor na comunidade) 31 .

Apesar disso, a eutanásia era realizada em todos os municípios estudados. No entanto, três coordenadores adotavam critérios para eutanásia divergentes do preconizado pelo PVCLV da seguinte forma: animal sintomático sem exames confirmatórios (dois municípios); somente uma sorologia reagente (um município); e exames mais específicos positivos, usando material coletado de punção de linfonodos e medula óssea para animais reagentes (um município). Os motivos relatados para essas divergências foram desde a falta de recursos materiais e humanos para a realização da eutanásia até controvérsias técnicas entre clínicos e poder público.

Outro fator considerado pelos coordenadores como razão da pouca efetividade da eutanásia como medida de controle da LV foi a demora entre o resultado reagente e a eutanásia, mantendo cães potencialmente transmissores da doença nas comunidades (Tabela 3). Essa demora teve como causas tanto a falta de recursos humanos (pessoal capacitado e em número adequado) e materiais (anestésicos, por exemplo) como a não aceitação dos proprietários de animais (como exemplos: uso de medidas judiciais e/ou proprietários que esconderam os animais).

É importante ressaltar que todos os coordenadores relataram que essa demora ocorre para animais oligossintomáticos e/ou assintomáticos, uma vez que a eutanásia tem melhor aceitação quando o bem-estar do animal está comprometido. De acordo com o citado em vários estudos, cães reagentes podem atuar como bons reservatórios, com grande poder de infectar o vetor da doença, mesmo não apresentando sinais clíni-
Tabela 3

Tempo decorrido entre os resultados reagentes de sorologia de cães e a eutanásia, segundo os coordenadores de programa entrevistados em seis municípios brasileiros. Brasil, 2013.

\begin{tabular}{llc}
\hline Tempo decorrido & \multicolumn{2}{c}{ Frequência de } \\
& resposta \\
& n & $\%$ \\
\hline Mais de um mês & 3 & 50,0 \\
Entre uma semana e um mês & 2 & 33,3 \\
Menos de uma semana & 1 & 16,7 \\
Total & 6 & 100,0 \\
\hline
\end{tabular}

Fonte: questionários semiestruturados.

cos 32,33,34. Assim, quanto mais tempo o animal é mantido no ambiente, maior a chance de transmitir a parasitose aos vetores e maior o risco de transmissão da doença ao homem 26,27,35.

Todos os coordenadores relataram terem tido dificuldades na condução das diretrizes do PVCLV também por interferências de clínicos veterinários. Disseram que é comum clínicos orientarem os proprietários de animais a recusarem as medidas de controle da LV, citando como justificativas: a falta de credibilidade dos exames realizados pela Secretaria de Saúde; a discordância do programa brasileiro com o resto do mundo; medidas alternativas como o tratamento canino, a vacinação contra LV e o uso de coleira em detrimento da eutanásia. Portanto, alguns clínicos veterinários colaboraram para aumentar ainda mais a desconfiança em relação ao controle de LV nos municípios, segundo os entrevistados.

Nenhum dos coordenadores de programa citaram parcerias positivas com ONGs protetoras de animais. A declaração de um dos coordenadores foi: "não existe a menor possibilidade de diálogo com essas entidades”. Os principais problemas referidos foram: utilização de apelo sentimental junto à população; convencimento de vereadores, deputados, prefeitos, ministério público, entre outros, quanto à ineficácia do programa, levando questão técnica para o âmbito político; difamação dos profissionais de saúde e divulgação de medidas contrárias ao PVCLV em redes sociais e meios de comunicação.

Sendo a eutanásia canina um tema polêmico e de pouca aceitação pública, tornou-se cada vez mais frequente a interferência do Poder Judiciário e político-partidário nesta questão que, primariamente, seria de competência do Poder Executivo. No contexto de interferências políticas, vale destacar a crescente luta da sociedade 
em prol dos direitos dos animais e pelo estabelecimento de políticas públicas para a sua defesa e proteção. Essa causa vem crescendo consideravelmente na sociedade e constitui mais um elemento que dificulta a eutanásia canina 36,37.

Assim, embora a eutanásia de cães reagentes sorologicamente esteja preconizada na Norma editada, na prática, controvérsias sobre este tema levam a recusas da comunidade e à demora para a execução desta medida podendo, desta forma, ser mais um fator para a expansão da $\mathrm{LV}$ no Brasil.

\section{- Medidas referentes ao vetor}

O controle vetorial, segundo o PVCLV, difere de acordo com o estrato epidemiológico do município. Naqueles somente com transmissão canina ou em áreas classificadas como de transmissão esporádica da doença, não é preconizado o controle químico. Sendo assim, no contexto epidemiológico de Campinas e Goiânia preconiza-se a eliminação canina sem a eliminação do inseto adulto. Ou seja, apesar do ciclo de transmissão da doença só ser mantido diante da presença do vetor, as medidas são centralizadas no controle do reservatório canino ${ }^{38}$. Estudos enfocando a estratégia de eliminação canina têm oferecido resultados conflitantes quando esta é utilizada separadamente do controle vetorial 22 , uma vez que a infecção de cães pode preceder a infecção humana e as chances são maiores em áreas com altas taxas de prevalência da infecção canina e presença do vetor 7 .

Assim, para os municípios somente com transmissão canina as medidas de controle vetorial são baseadas em ações de manejo ambiental que visam a reduzir condições favoráveis para o estabelecimento de criadouros do vetor (presença de matéria orgânica e de locais sombreados). Tanto em Campinas quanto em Goiânia, foi relatada a dificuldade da realização de manejo ambiental como preconizado pelo PVCLV, uma vez que as áreas de transmissão são periurbanas, com a presença de vegetação abundante dentro das casas e nas áreas comuns, dificultando a sua remoção, seja pela falta de adesão dos moradores ou pela insuficiência de recursos das prefeituras para esta atividade.

Além de difícil, estudos mostram que não há evidências de que o manejo ambiental tenha impacto nas fases imaturas do inseto, já que são pouco conhecidos os locais de procriação natural e de descanso diurno dos flebotomíneos 38 . Por esse fato, o controle do inseto é dependente do combate aos insetos adultos 39 .

O inseticida preconizado pelo PVCLV para municípios com transmissão humana é um pire- troide borrifado em toda a extensão das paredes do intradomicílio e peridomicílio das residências. As entrevistas dos coordenadores de programa de Fortaleza, Bauru, Campo Grande e Belo Horizonte mostram que a realização de controle químico adequado do vetor é um dos maiores desafios encontrados pelo programa devido a fatores como: descontinuidade da ação por falta de recursos materiais e/ou humanos; pela recusa da comunidade; e por ser caro e complexo. Ou seja, houve relato de intermitência dessa atividade com maiores falhas quanto maior a extensão territorial dos municípios e maior número de imóveis a serem borrifados. E ainda relatos de que quando a atividade é realizada, ocorre de forma limitada uma vez que há recusas significativas das comunidades tanto de forma parcial (o proprietário não permite a borrifação do interior do domicílio) como total (o proprietário não autoriza a borrifação do intradomicílio nem do peridomicílio) 40. Tais limitações na aplicação química têm levado à redução do uso de inseticidas para o controle vetorial nos últimos anos, em alguns dos municípios pesquisados, sendo mais um dos fatores que podem contribuir para que o número de casos humanos e caninos da enfermidade se mantenha elevado.

\section{- Medidas referentes à educação em saúde}

Tradicionalmente, ações de educação em saúde estão inseridas em todo programa de controle de doenças sob vigilância no Brasil. O PVCLV enfatiza que a educação em saúde deve estar incluída em todos os serviços que desenvolvem ações de controle de LV, sendo imprescindível a capacitação das equipes de saúde e de educadores, o esclarecimento da população e o estabelecimento de parcerias com os setores público e privado 7 .

Embora seja atribuído um papel de grande relevância às atividades de educação em saúde, elas são pouco valorizadas dentro do contexto dos serviços de vigilância em saúde. Isso fica evidenciado pelo fato de nenhum dos municípios analisados contar com profissionais da área de educação em suas equipes, já que o pressuposto na maioria das vezes é que tais ações sejam executadas pelos próprios técnicos que não dispõem de formação nesta área e que estão também envolvidos na eliminação dos cães infectados, gerando descrédito e oposição ao invés da colaboração das comunidades.

No geral, as ações de educação em saúde têm caráter informativo que visam esclarecer sobre as formas de prevenção e controle da doença, estimulando a posse responsável de animais e medidas de manejo ambiental, com campanhas de caráter informativo que enfatizam a doença e 
seus perigos. Como meio de atingir a comunidade, coordenadores recorrem à entrega de folhetos educativos, conversa casa a casa e algumas palestras para as comunidades. Recorrem também à imprensa, porém não de maneira unânime; quatro coordenadores relataram que os órgãos de comunicação colaboram na educação da população ao darem espaço aos técnicos para se posicionarem, e dois coordenadores citaram a imprensa como elemento de discórdia como mostra a afirmação de um deles: "parei de ir à imprensa pelo fato de só ser divulgado o lado ruim do programa".

Para avaliar como as abordagens citadas estavam sendo efetivas, houve questionamento sobre a mudança de comportamento da população após tais atividades educativas. Como resposta, quatro coordenadores relataram ocorrer menos de $50 \%$ de adesão e anuência às propostas do programa, corroborando o estudo realizado em Belo Horizonte que mostrou que o nível de conhecimento da população sobre LV se restringe a informações superficiais que não possibilitam a contribuição ativa e permanente da população 41 .

Em suma, as atividades de educação em saúde no PVCLV desses municípios não têm alcançado sucesso, seja na comunicação de risco, seja na mobilização social e, como resultado, há pouco envolvimento da comunidade na prevenção e controle da doença.

\section{Considerações finais}

As entrevistas realizadas com os coordenadores de programas de grandes cidades brasileiras com transmissão de LV estudadas mostraram inúmeras dificuldades na execução das atividades do programa de controle e prevenção. Em nenhum dos municípios pesquisados, independentemente do estrato epidemiológico, há cumprimento da totalidade das atividades preconizadas pelo PVCLV, seja por dificuldades de caráter estrutural das prefeituras, seja por resistência dos sujeitos implicados com a doença.

De forma que as dificuldades encontradas neste estudo podem ser ponto de partida para a reflexão sobre a necessidade de revisão do PVCLV, dada a impossibilidade de cumprimento pleno de suas diretrizes no âmbito municipal. Tal revisão deveria considerar a flexibilização de algumas diretrizes, respeitando as singularidades e as especificidades do território e da população atingida, bem como as demandas da sociedade, particularmente a relação de afeto do homem com o seu animal de estimação. Além disso, deveria ampliar o compromisso do Estado com a garantia de recursos humanos, materiais e financeiros para a continuidade das atividades propostas, incluindo investimento no treinamento das equipes de saúde, em ação comunicativa que melhorasse o diálogo com os grupos sociais atingidos pelo agravo.

Algumas limitações deste estudo podem ser destacadas: as opiniões de somente seis coordenadores municipais entrevistados podem não refletir a experiência de outros no restante do país, bem como o fato de as entrevistas terem sido realizadas somente com os coordenadores (trazendo o posicionamento da gestão, nem sempre compartilhada por toda a equipe envolvida). Entretanto, deve-se considerar que a LV é uma doença de grande magnitude nos municípios observados, e que houve bastante coerência e semelhanças nas experiências relatadas pelos gestores entrevistados em diferentes regiões brasileiras.

\section{Colaboradores}

A. P. B. von Zuben contribuiu com a concepção, coleta de dados e elaboração do artigo. M. R. Donalísio contribuiu com a concepção, elaboração e revisão do artigo.

\section{Agradecimentos}

Aos coordenadores de programa entrevistados. 


\section{Referências}

1. Dantas-Torres F. Canine leishmaniosis in South America. Parasit Vectors 2009; 2 Suppl 1:S1.

2. Harhay MO, Olliaro PL, Costa DL, Costa CH. Urban parasitology: visceral leishmaniasis in Brazil. Trends Parasitol 2011; 27:403-9.

3. World Health Organization. Control of the leishmaniases: report of a meeting of the WHO Expert Committee on the Control of Leishmaniases. Geneva: World Health Organization; 2010. (WHO Technical Report Series, 949).

4. Alvar J, Vélez ID, Bern C, Herrero M, Desjeux P, Cano J, et al. Leishmaniasis worldwide and global estimates of its incidence. PLoS One 2012; 7:e35671.

5. Marcondes M, Rossi CN. Leishmaniose visceral no Brasil. Braz J Vet Res Anim Sci 2013; 50:341-52.

6. Werneck GL. Expansão geográfica da leishmaniose visceral no Brasil. Cad Saúde Pública 2010; 26: 644-5.

7. Ministério da Saúde. Manual de vigilância e controle da leishmaniose visceral. Brasília: Ministério da Saúde; 2006.

8. Secretaria de Vigilância em Saúde, Ministério da Saúde. Casos confirmados de leishmaniose visceral, Brasil. Grandes regiões e Unidades Federadas, 1990 a 2010. Brasília: Ministério da Saúde; 2013.

9. Rangel O, Hiramoto RM, Henriques LF, Taniguchi HH, Ciaravolo RMC, Tolezano JE, et al. Classificação epidemiológica dos municípios segundo o Programa de Vigilância e Controle da Leishmaniose Visceral Americana no Estado de São Paulo, para 2013. BEPA. Boletim Epidemiológico Paulista 2013; 10:5-16.

10. Azevedo EMR, Duarte SC, Costa HX, Alves CEF, Silveira Neto OJ, Jayme VS, et al. Estudo da leishmaniose visceral canina no Município de Goiânia, Goiás, Brasil. Rev Patol Trop 2011; 40:159-68.

11. von Zuben APB, Angerami RN, Castagna C, Baldini MDB, Donalisio MR. The first canine visceral leishmaniasis outbreak in Campinas, State of São Paulo Southeastern Brazil. Rev Soc Bras Med Trop 2014; 47:385-8.

12. Brazuna JCM, Silva EA, Brazuna JM, Domingos IH, Chaves N, Honer MR, et al. Profile and geographic distribution of reported cases of visceral leishmaniasis in Campo Grande, State of Mato Grosso do Sul, Brazil, from 2002 to 2009. Rev Soc Bras Med Trop 2012; 45:601-6.

13. Secretaria Municipal de Saúde Pública, Prefeitura Municipal de Campo Grande. Plano Municipal de Saúde de Campo Grande. Campo Grande: Prefeitura Municipal de Campo Grande; 2013.

14. Secretaria Municipal de Saúde, Prefeitura Municipal de Belo Horizonte. Vigilância epidemiológica da leishmaniose visceral em Belo Horizonte. Belo Horizonte: Prefeitura Municipal de Belo Horizonte; 2014.

15. Lopes EG, Magalhães DF, Silva JA, Haddad JPA, Moreira EC. Distribuição temporal e espacial da leishmaniose visceral em humanos e cães em Belo Horizonte - MG, 1993 a 2007. Arq Bras Med Vet Zootec 2010; 62:1062-71.

16. Lima MB, Batista EAR. Epidemiologia da leishmaniose visceral humana em Fortaleza - CE. Rev Bras Promoç Saúde (Impr) 2009; 22:16-23.
17. Secretaria Municipal de Saúde, Prefeitura Municipal de Fortaleza. Boletim epidemiológico: leishmaniose visceral. Fortaleza: Prefeitura Municipal de Fortaleza; 2013.

18. Secretaria Municipal de Saúde, Prefeitura Municipal de Bauru. Leishmaniose visceral: atualização epidemiológica. Bauru: Prefeitura Municipal de Bauru; 2012.

19. Souza VAF, Cortez LRPB, Dias RA, Amaku M, Ferreira Neto JS, Kuroda RBS, et al. Space-time cluster analysis of American visceral leishmaniasis in Bauru, São Paulo State, Brazil. Cad Saúde Pública 2012; 28:1949-64.

20. Tauil PL. Perspectivas de controle de doenças transmitidas por vetores no Brasil. Rev Soc Bras Med Trop 2006; 39:275-7.

21. Dujardin JC, Campino L, Cañavete C, Dedet J-P, Gradoni L, Soteriadou K, et al. Spread of vectorborne diseases and neglect of leishmaniasis, Europe. Emerg Infect Dis 2008; 14:1013-8.

22. Gontijo CMF, Melo MN. Leishmaniose visceral no Brasil: quadro atual, desafios e perspectivas. Rev Bras Epidemiol 2004; 7:338-49.

23. Silva Júnior JB. Epidemiologia em serviço: uma avaliação de desempenho do Sistema Nacional de Vigilância em Saúde [Tese de Doutorado]. Campinas: Faculdade de Ciências Médicas, Universidade Estadual de Campinas; 2004.

24. Alves WA. Controle da leishmaniose visceral baseado no reservatório canino. In: Informe final de la Reunión de Expertos OPS/OMS sobre Leishmaniasis Visceral en las Américas. Washington DC: Organización Panamericana de la Salud; 2006. p. 94-8.

25. Palatnik-de-Sousa CB, Day MJ. One health: the global challenge of epidemic and endemic leishmaniasis. Parasit Vectors 2011; 4:1-10.

26. Costa CHN. How effective is dog culling in controlling zoonotic visceral leishmaniasis? A critical evaluation of the science, politics and ethics behind this public health policy. Rev Soc Bras Med Trop 2011; 44:232-42.

27. Romero GAS, Boelaert M. Control of visceral leishmaniasis in Latin America - systematic review. PLoS Negl Trop Dis 2010; 4:e584.

28. Quinnell RJ, Courtenay O. Transmission, reservoir hosts and control of zoonotic visceral leishmaniasis. Parasitology 2009; 136:1915-34.

29. Conselho Federal de Medicina Veterinária. Resolução no 1.000, de 11 de maio de 2012. Diário Oficial da União 2012; 17 mai.

30. Dantas-Torres F, Solano-Gallego L, Baneth G, Ribeiro VM, de Paiva-Cavalcanti M, Otranto D. Canine leishmaniosis in the Old and New Worlds: unveiled similarities and differences. Trends Parasitol 2012; 28:531-8.

31. Coura-Vital W, Ker HG, Roatt BM, Aguiar-Soares RD, Leal GG, Moreira ND, et al. Evaluation of change in canine diagnosis protocol adopted by the visceral leishmaniasis control program in Brazil and a new proposal for diagnosis. PLoS One 2014; 9:e91009.

32. Moreno J, Alvar J. Canine leishmaniasis: epidemiological risk and the experimental model. Trends Parasitol 2002; 18:399-405. 
33. Silva AVM, Paula AA, Cabrera MAA, Carreira JCA. Leishmaniose em cães domésticos: aspectos epidemiológicos. Cad Saúde Pública 2005; 21:324-8.

34. Chaves MM, Teixeira MJ, Pompeu MML, Sousa RN, Lima JWO. Evaluation of the potential reservoir dog in visceral leishmaniasis in two municipalities of the State of Ceara. PUBVET 2013; 7:1568

35. Belo VS, Werneck GL, Barbosa DS, Simões TC, Nascimento BWL, Silva ES, et al. Factors associated with visceral leishmaniasis in the americas: a systematic review and meta-analysis. PLoS Negl Trop Dis 2013; 7:e.2182.

36. Junior VLP, Lane VFM, Pereira LRM. Judicialização das ações de vigilância em saúde: o caso da leishmaniose visceral. Tempus Actas de Saúde Coletiva 2013; 7:55.

37. Pereira LRM. Atuação do Ministério Público direcionada ao Programa de Vigilância da Leishmaniose Visceral no contexto das ações de controle do reservatório da espécie canina [Dissertação de Mestrado]. Recife: Centro de Pesquisas Aggeu Magalhães, Fundação Oswaldo Cruz; 2010.
38. Feliciangeli MD. Natural breeding places of phlebotomine sandflies. Med Vet Entomol 2004; 18: 71-80.

39. Amóra SSA, Bevilaqua CML, Feijó FMC, Alves ND, Maciel MV. Control of phlebotomine (Diptera: Psychodidae) leishmaniasis vectors. Neotrop Entomol 2009; 38:303-10.

40. Santana Filho FC, Silva JA, Magalhães DF, Meneses JNC, Haddad JPA, Morais MHF, et al. Refusal of spraying of buildings and the occurrence of cases of visceral leishmaniasis in North West region of Belo Horizonte, Brazil. Arq Bras Med Vet Zootec 2012; 64:899-908.

41. Borges BKA, Silva JA, Haddad JPA, Moreira EC, Magalhães DF, Ribeiro LML, et al. Avaliação do nível de conhecimento e de atitudes preventivas da população sobre a leishmaniose visceral em Belo Horizonte, Minas Gerais, Brasil. Cad Saúde Pública 2008; 24:777-84.

\begin{abstract}
The expansion of visceral leishmaniasis (VL) transmission areas in Brazil raises questions concerning the country's control strategies. The guidelines of the Brazilian Visceral Leishmaniasis Control Program (PVCLV) focus on decreases in morbidity and case-fatality, reservoir and vector control, and health education. This article assesses difficulties in implementing the measures recommended by the PVCLV, according to the program's coordinators in large cities with canine and/or human transmission (Campinas, Bauru, Goiânia, Campo Grande, Fortaleza, and Belo Horizonte). Semi-structured interviews identified the following main problems: discontinuity of control activities, resistance to canine euthanasia by dog owners, and low coverage of chemical vector control. Conclusion: the interviews revealed numerous obstacles to implementation of the measures proposed by the PVCLV and a clear need to reassess Brazil's VL policy.
\end{abstract}

Visceral Leishmaniasis; Disease Prevention; Health Programs and Plans

\section{Resumen}

La expansión de las áreas de transmisión de leishmaniasis visceral (LV) plantea cuestiones sobre las estrategias de control empleadas en Brasil. Las directrices del Programa Brasileño de Control de la Leishmaniasis Visceral (PVCLV) están centradas en la disminución de la morbilidad y letalidad, control de reservorios y vectores, mediante la educación en salud. Este artículo procuró evaluar las dificultades en la ejecución de las acciones preconizadas por el PVCLV, según los coordinadores de este programa, en municipios brasileños de grandes dimensiones, donde ha existido transmisión canina y/o humana (Campinas, Bauru, Goiânia, Campo Grande, Fortaleza y Belo Horizonte). Se realizaron entrevistas semiestructuradas, siendo los siguientes los principales problemas identificados: discontinuidad de las actividades de control, resistencia por parte de los propietarios de perros con indicación de eutanasia y baja cobertura de control químico. Como conclusión, las entrevistas mostraron que factores innumerables impiden el cumplimiento de las actividades propuestas por el PVCLV, quedando clara la necesidad de una reevaluación de la política brasileña sobre la $L V$.

Leishmaniasis Visceral; Prevención de Enfermedades; Planes y Programas de Salud
Recebido em 07/Jun/2015

Versão final reapresentada em 14/Set/2015

Aprovado em 28/Out/2015 